Variants of early-onset restrictive eating disturbances in middle childhood

Susanne Kurz, M.D. ${ }^{\text {ab,c*, }}$, Zoé van Dyck, M.D. ${ }^{\text {, }}$, Daniela Dremmel, M.D. ${ }^{a}$, Simone Munsch, PhD. ${ }^{\mathrm{a}}$, Anja Hilbert, PhD. ${ }^{\mathrm{b}, \mathrm{c}}$

This is the peer reviewed version of the following article: Variants of early-onset restrictive eating disturbances in middle childhood, which has been published in final form at http://dx.doi.org/ 10.1002/eat.22461. This article may be used for non-commercial purposes in accordance with Wiley Terms and Conditions for Self-Archiving. [Link to: http://olabout.wiley.com/WileyCDA/Section/id-828039.html]

${ }^{\text {a }}$ Department of Psychology, University of Fribourg, Rue P.-A. de Faucigny 2, Fribourg, Switzerland

${ }^{\mathrm{b}}$ Integrated Research and Treatment Center AdiposityDiseases, University of Leipzig, Medical Center, Philipp-Rosenthal-Strasse 27, Leipzig, Germany

${ }^{\mathrm{c}}$ Department of Medical Psychology and Medical Sociology, University of Leipzig, Medical Center, Leipzig, Germany

${ }^{\mathrm{d}}$ Institute for Health and Behavior, Research Unit INSIDE, University of Luxembourg, Campus Walferdange, Luxemburg

* Corresponding author. University of Fribourg, Department of Psychology, Rue P.-A. de Faucigny 2, CH-1700 Fribourg, Switzerland. Phone: +41 26300 7352, Email: susanne.kurz@unifr.ch. 
This research was supported by the Swiss National Science Foundation (SNSF) (grant number 100014132045 /1) and by the German Federal Ministry of Education and Research (BMBF) (grant number 01EO1001).

Submitted: $29^{\text {th }}$ of June 2015

\begin{abstract}
Objective: This study sought to determine the factor structure of the newly developed self-report screening questionnaire Eating Disturbances in Youth-Questionnaire (EDY-Q) as well as to report the distribution of variants of early-onset restrictive eating disturbances characteristic of avoidant/restrictive food intake disorder (ARFID) in a middle childhood population sample. Method: Using the EDY-Q, a total of 1444 children aged 8-13 years were screened in elementary schools in Switzerland via self-report. The factor analysis of the 12 items covering ARFID related symptoms was performed using a principal component analysis (PCA). Results: The PCA showed a four factor solution, with clear allocation to the scales covering three variants of early-onset restrictive eating disturbances and weight problems. Inadequate overall food intake was reported by $19.3 \%$ of the children, a limited accepted amount of food by $26.1 \%$, and food avoidance based on a specific underlying fear by $5.0 \%$. Discussion: The postulated factor structure of the EDY-Q was confirmed, further supporting the existence of distinct variants of early-onset restrictive eating disturbances. Avoidant/restrictive eating behavior seems to be a common experience in middle childhood, but results have to be confirmed using validated interviews.
\end{abstract}

Keywords: early-onset restrictive eating disturbances, food avoidance emotional disorder, selective eating, functional dysphagia, prevalence 
Word count: 186

\section{VARIANTS OF EARLY-ONSET RESTRICTIVE EATING DISTURBANCES IN MIDDLE CHILDHOOD}

The new DSM-5 eating disorder diagnosis Avoidant/Restrictive Food Intake Disorder (ARFID) captures avoidant or restrictive eating disturbances without shape or weight concerns. ${ }^{1}$ Existing terms describing these eating disturbances can be categorized in terms of an inadequate overall food intake, the limited amount of accepted food as well as food avoidance associated with an underlying specific fear. ${ }^{2}$ These primarily middle childhood disturbances can go along with significant weight loss and/or growth impairment. ${ }^{1}$ Contrary to anorexia or bulimia nervosa, they are unrelated to shape or weight concerns.,4

An inadequate overall food intake, called food avoidance emotional disorder (FAED), ${ }^{5}$ is an emotional disturbance with food avoidance as a prominent symptom, ${ }^{6}$ which has to persist for at least one month without organic cause. ${ }^{7}$ Children with FAED encounter emotional problems, such as sadness or anxiety, ${ }^{2,8}$ that interfere with their food intake. ${ }^{2}$ Health problems frequently co-occur, ${ }^{9}$ and prognosis for psychological outcomes is worse than for emotional disorders. ${ }^{6}$ A common term describing limited food intake is selective eating (SE), consisting of eating a highly limited range of foods of a particular color, texture, or brand. ${ }^{10,11}$ While selective eaters accept a very limited range of foods, picky/choosy/fussy eaters ${ }^{11,12}$ have a range of foods that they will not eat. SE and related forms are normal developmental behaviors in toddlers, but if persistent, the child's and parent's well-being may 
be impacted negatively. ${ }^{2-4}$ Further, food avoidance based on an underlying specific fear, called food phobia, can present as fear of choking or swallowing (functional dysphagia; FD) a commonly cited food phobia -, fear of vomiting (emetophobia), fear of contamination, and fear of harmful foods (e.g., regarding cholesterol), likely preceded by traumatic experiences. These children are often underweight and physically unwell. ${ }^{2,9}$ While FAED is considered as distinct food intake disorder, SE and FD represent behavioral syndromes which can be part of other disorders. ${ }^{3,8,13}$

Few epidemiological studies on the variants of early-onset restrictive eating disturbances exist, one of which identified $29.0 \%$ of the 86 8- to 18-year old early-onset eating disordered patients with FAED, 17.0\% with SE and 1.0\% with FD applying the Great Ormond Street criteria (Appendix A; $\left.{ }^{14}\right) .{ }^{15}$ Further, in a study of 1326 5- to 7-year-old healthy children $7.3 \%$ were reported to be picky eaters and $1.7 \%$ slow/poor eaters using validated questionnaires. ${ }^{16}$ FAED, SE, FD frequently co-occur, ${ }^{3,17}$ but numbers are still unknown. FAED seems to be more common in girls than in boys, ${ }^{6,18}$ whereas SE and related terms tend to be more common in boys than in girls. ${ }^{9,15,19,20}$ Nothing is known about the gender distribution in food phobias.

The epidemiology of the variants of early-onset restrictive eating disturbances in the general non-clinical population remains unclear. Early detection, however, is essential for preventing a chronic course ${ }^{21,22}$ and promoting a healthy development. ${ }^{23,24}$ To date, no standardized assessment exists for capturing early-onset restrictive eating disturbances.

This study sought to examine the factor structure of the Eating Disturbances in YouthQuestionnaire (EDY-Q; ${ }^{25}$ ), a newly developed screening instrument for identifying earlyonset restrictive eating disturbances, and to report the distribution of their variants in middle childhood. A four-factor solution of the EDY-Q was hypothesized including FAED, SE, FD, and weight problems. ${ }^{25}$ 


\section{METHOD}

\section{$\underline{\text { Participants }}$}

Children $(N=1444)$ were screened in primary schools $\left(3^{\text {rd }}\right.$ to $6^{\text {th }}$ grade $)$ in three cities of Switzerland and surrounding areas in German (47.2\%) and French (52.8\%) using the EDY$\mathrm{Q}^{25,26}$ (Appendix A). Children were on average $10.55(S D=1.89)$ years old (range 8-13 years), and 53.9\% were girls (779/1444). Based on self-report data, a total of $13.0 \%(N=$ $187 / 1356$; body mass index $(\mathrm{BMI})<10^{\text {th }}$ percentile) were underweight, $75.3 \%(N=$ $1087 / 1356 ; 10^{\text {th }}-90^{\text {th }}$ BMI percentile $)$ were normal weight, and $5.7 \%(N=82 / 1356$; $\mathrm{BMI}>$ $90^{\text {th }}$ BMI percentile) were overweight or obese according to current internationally comparable guidelines. ${ }^{27,28}$

\section{$\underline{\text { Measures }}$}

The EDY-Q includes 12 items based on DSM-5 criteria for ARFID and its variants using the Great Ormond Street criteria and related literature. Items were rated on a 7-point Likert scale (never $=0$; always $=6$ ). Previous results on this study's sample revealed good psychometric properties with low missing data (Appendix A). ${ }^{26}$ To date, no findings on the concordance with parent/clinician report exist.

\section{$\underline{\text { Statistical Analysis }}$}

A principal component analysis (PCA) with orthogonal VARIMAX rotation was used to determine the factor structure of the EDY-Q (Kaiser-Guttman extraction ${ }^{29}$ ). For practical significance, the cut-off for factor loadings was set at $\geq .40 .{ }^{30}$ Cronbach's $\alpha$ and mean interitem correlations were used to determine internal consistency and homogeneity of extracted factors.

Distribution of early-onset restrictive eating disturbances was determined using cut-off criteria and dichotomization of items (Appendix A). Variations of early-onset restrictive eating disturbances by gender, age, or weight status were tested using $\chi^{2}$ or Kruskal-Wallis 
tests and post-hoc analyses with Bonferroni corrections. The overall $p$ value was set at $<.05$ (two-tailed).

\section{RESULTS}

\section{Exploratory Factor Analysis}

Conditions for the PCA were met (Kaiser-Meyer-Olkin $=.71$, Bartlett's $p<0.01$ ). As hypothesized, the PCA resulted in a four-factor solution, which accounted for $57.3 \%$ of variance (Table 1). After rotation, the first (SE) and second (FAED) factor accounted for 15.5\% of variance each, the third (Weight Problems) factor accounted for $13.3 \%$, and the fourth factor (FD) accounted for $13.1 \%$ of variance.

\section{Reliability}

Homogeneity of subscales was low to medium with an inter-item correlation of $.19 \leq r$ $\leq .39$. Internal consistencies for the four factors were low $(.42 \leq$ Cronbach's $\alpha \leq .52)$. Correlations between subscales were low, but significant $(p<0.01 ; .17 \leq r \leq .26){ }^{31}$

\section{$\underline{\text { Prevalence }}$}

Overall, 36.1\% (521/1444) of the children reported symptoms of at least one earlyonset restrictive eating disturbance. SE was most commonly reported in 26.1\% (377/1444) of children, followed by FAED in 19.3\% (278/1444), and FD in 5.0\% (72/1444). There was overlap among variants in 9.3\% (134/1444) of children for FAED and SE, in 3.1\% (45/1444) for SE and FD, and in 2.9\% (42/1444) for FAED and FD. A total of 2.3\% (33/1444) of children reported symptoms of all three variants.

Boys and underweight children reported SE significantly more often than girls and normal- or overweight children (Table 2). Younger children were significantly more likely to report FAED and FD than older children. 


\section{DISCUSSION}

The postulated factor structure of the screening instrument EDY-Q was reproduced with three factors covering FAED, SE, and FD, and one factor covering weight problems. ${ }^{9,32}$ The three early-onset restrictive eating disturbances were commonly reported by children aged 8 to 13 years.

SE was reported in $26.1 \%$ of children, FAED in $19.3 \%$, and FD in $5.0 \%$. In a previous study with the same sample 3.2\% (46/1444) of the children reported ARFID features within which $60.9 \%$ reported SE, 39.1\% FAED, and $15.2 \%$ FD. ${ }^{26}$ Therefore, variants of early-onset restrictive eating disturbances present within the diagnosis of ARFID ${ }^{26}$ but they emerged more frequently without considering ARFID criteria. Further, the results on SE were in line with previous studies, indicating mother-reported SE in $13.0 \%$ to $29.0 \%$ of children $2-18$ years of age. ${ }^{10,11,21}$ No comparable studies were identified on the prevalence of FAED and FD. In a population-based study with 5-7-year old children, emotional undereating was reported by mothers in $26.0 \%$ of the children, ${ }^{16}$ similar to our finding for FAED.

The results further showed that the three presentations can coexist, which was also reported in clinical observations. ${ }^{3,17}$ The relatively high comorbidity rates leave the question, whether or not it is possible to differentiate among these disturbances. However, as the PCA showed, the variants FAED, SE, and FD were factor-analytically determined in the EDY-Q, supporting their existence and uniqueness.

Boys indicated SE more often than girls, which coincides with previous studies in which over two-thirds of the selective eaters were boys. ${ }^{6,9}$ No studies exist corroborating our finding that younger children (7-10 years) were significantly more likely to report FAED and FD than older children. Possibly, younger children had more difficulty understanding eating disorder symptoms ${ }^{33}$ and therefore underestimated their seriousness. Alternatively, these eating disturbances may be more common at a younger age. Our finding that underweight 
children reported SE more often than normal and overweight children matched the diagnostic criteria of ARFID, which is required to create serious clinical consequences (e.g., weight loss, growth impairment). ${ }^{1,32,34}$ Further studies should focus on long-term general and eating disorder outcomes of early-onset restrictive eating disturbances.

Regarding study limitations, self-report questionnaires are less accurate than expert interviews and may result in overestimation of prevalence rates. ${ }^{35}$ Nevertheless, self-report questionnaires assessing eating disturbances in childhood can obtain valid information. ${ }^{36,37}$ Further, weight status might be affected by biased reporting of weight and height and should be interpreted with caution. ${ }^{38-40}$ Because of low number of items per subscale internal consistencies of the EDY-Q were expectedly low. ${ }^{41}$ In this case, the use of the mean interitem correlation as a measure for reliability is recommended, ${ }^{42}$ which was satisfactory. ${ }^{31,41,42}$

Overall, this study provides initial epidemiological information supporting the variants of early-onset restrictive eating disturbances in middle childhood. The large community-based sample size, with German- and French-speaking children, appears supportive of the instrument's generalizability. The validity of the EDY-Q as a screening instrument for earlyonset restrictive eating disturbances will benefit from further confirmatory studies.

Word count: 1498 


\section{References}

1. American Psychiatric Association. Diagnostic and statistical manual of mental disorders: DSM-5. 5th ed. Arlington, VA: American Psychiatric Association; 2013.

2. Bryant-Waugh R, Markham L, Kreipe RE, Walsh BT. Feeding and eating disorders in childhood. Int J Eat Disord 2010;43:98-111.

3. Nicholls D, Bryant-Waugh R. Eating disorders of infancy and childhood: definition, symptomatology, epidemiology, and comorbidity. Child Adolesc Psychiatr Clin N Am 2008;18:17-30.

4. Watkins B, Lask B. Eating disorders in school-aged children. Child Adolesc Psychiatr Clin N Am 2002;11:185-199.

5. Bryant-Waugh RJ, Piepenstock EHC. Childhood Disorders: Feeding and Related Disorders of Infancy or Early Childhood (3rd ed.). In: Tasman A, editor. Psychiatry. Chichester, England, Hoboken, NJ: Wiley-Blackwell, 2008, p. 830-846.

6. Higgs JF, Goodyer IM, Birch J. Anorexia nervosa and food avoidance emotional disorder. Arch Dis Child 1989;64:346-351.

7. Bryant-Waugh R, Kreipe RE. Avoidant/Restrictive Food Intake Disorder in DSM-5. Psychiatr Ann 2012;42:402-405.

8. Kreipe RE, Palomaki A. Beyond Picky Eating: Avoidant/Restrictive Food Intake Disorder. Curr Psychiatry Rep 2012;14:421-431. 
9. Bryant-Waugh R, Lask B. Overview of the Eating Disorders (3rd ed.). In: Lask B, BryantWaugh R, editors. Eating disorders in childhood and adolescence. London, New York: Routledge, 2007, p. 35-50.

10. Mascola AJ, Bryson SW, Agras WS. Picky eating during childhood: A longitudinal study to age 11years. Eat Behav 2010;11:253-257.

11. Jacobi C, Schmitz G, Agras WS. Is picky eating an eating disorder? Int J Eat Disord 2008;41:626-634.

12. Dovey TM, Staples PA, Gibson EL, Halford JCG. Food neophobia and 'picky/fussy' eating in children: A review. Appetite 2008;50:181-193.

13. Nicholls D, Chater R, Lask B. Children into DSM don't go. Int J Eat Disord 2000;28:317324.

14. Bryant-Waugh R, Lask B. Annotation: Eating Disorders in Children. J Child Psychol Psychiatry 1995;36:191-202.

15. Cooper PJ, Watkins B, Bryant-Waugh R, Lask B. The nosological status of early onset anorexia nervosa. Psychol Med 2002;32:873-880.

16. Micali N, Simonoff E, Elberling H, Rask CU, Olsen EM, Skovgaard AM. Eating Patterns in a Population-Based Sample of Children Aged 5 to 7 Years: Associations With Psychopathology and Parentally Perceived Impairment. J Dev Behav Pediatr 2011;32:572-580.

17. Bryant-Waugh R. Avoidant restrictive food intake disorder: An illustrative case example. Int J Eat Disord 2013;46:420-423.

18. Christie D, Bryant-Waugh R, Lask B, Gordon I. Neurobiological aspects of early onset eating disorders. In: Hoek HW, Treasure J, Katzman M, editors. Neurobiology in the treatment of eating disorders. Chichester, New York: Wiley, 1998, p. 292. 
19. Timimi S, Douglas J, Tsiftsopoulou K. Selective eaters: a retrospective case note study. Child Care Health Dev 1997;23:265-278.

20. Nicholls D, Christie D, Randall L, Lask B. Selective Eating: Symptom, Disorder or Normal Variant. Clin Child Psychol Psychiatry 2001;6:257-270.

21. Marchi M, Cohen P. Early childhood eating behaviors and adolescent eating disorders. J Am Acad Child Adolesc Psychiatry 1990;29:112-117.

22. Kotler LA, Cohen P, Davies M, Pine DS, Walsh BT. Longitudinal relationships between childhood, adolescent, and adult eating disorders. J Am Acad Child Adolesc Psychiatry 2001;40:1434-1440.

23. Bryant-Waugh R, Knibbs J, Fosson A, Kaminski Z, Lask B. Long term follow up of patients with early onset anorexia nervosa. Arch Dis Child 1988;63:5-9.

24. Rosen DS. Eating disorders in children and young adolescents: etiology, classification, clinical features, and treatment. Adolesc Med 2003;14:49-59.

25. van Dyck Z, Bellwald L, Kurz S, Dremmel D, Munsch S, Hilbert A. Essprobleme im Kindesalter: Screening in der allgemeinen Bevölkerung [Eating disturbances in childhood. Screening in the general popultation]. Zeitschrift für Gesundheitspsychologie 2013;21:91100.

26. Kurz S, van Dyck Z, Dremmel D, Munsch S, Hilbert A. Early-onset restrictive eating disturbances in primary school boys and girls. Eur Child Adolesc Psychiatry 2014. doi: 10.1007/s00787-014-0622-z.

27. Arbeitsgemeinschaft Adipositas im Kindes- und Jugendalter. Leitlinien der Arbeitsgemeinschaft Adipositas im Kindes- und Jugendalter [Guidelines of the Workgroup for Adiposity in Childhood and Adolescence]. [April 03, 2013]; Available from: http://www.adipositas-

gesellschaft.de/fileadmin/PDF/Leitlinien/AGA_S2_Leitlinie.pdf. 
28. Kuczmarski RJ, Ogden CL, Guo SS, et al. 2000 CDC growth charts for the United States: Methods and development. Vital Health Stat 2002;11:1-190.

29. Hakstian AR, Roger WT, Cattell RB. The Behavior of Number-Of-Factors Rules with Simulated Data. Multivar Behav Res 1982;17:193-219.

30. Stevens J. Applied multivariate statistics for the social sciences. 5th ed. New York: Routledge; 2009.

31. Clark LA, Watson D. Constructing validity: Basic issues in objective scale development. Psychol Assess 1995;7:309-319.

32. Al-Adawi S, Bax B, Bryant-Waugh R, Claudino AM, Hay P, Monteleone P et al. Revision of ICD - status update on feeding and eating disorders. Advances Eat Disord 2013;1:10_ 20.

33. Braet C, van Strien T. Assessment of emotional, externally induced and restrained eating behaviour in nine to twelve-year-old obese and non-obese children. Behav Res Ther 1997;35:863-873.

34. Kenney L, Walsh T. Avoidant/Restrictive Food Intake Disorder (ARFID): Defining ARFID. [February 04, 2014]. Available from: http://eatingdisordersreview.com/nl/nl edr $\underline{24} 3$ 1.html

35. Fairburn CG, Beglin SJ. Assessment of eating disorders. Int J Eat Disord 1994;16:363370.

36. van Strien T, Oosterveld P. The children's DEBQ for assessment of restrained, emotional, and external eating in 7- to 12-year-old children. Int J Eat Disord 2008;41:72-81.

37. Hilbert A, Hartmann AS, Czaja J. Child Eating Disorder ExaminationQuestionnaire: Evaluation der deutschsprachigen Version des Essstörungsfragebogens für Kinder [Evaluation of the german-speaking version of the child eating disorder examination-questionnaire]. Klinische Diagnostik und Evaluation 2008;1:447-464. 
38. Seghers J, Claessens AL. Bias in Self-Reported Height and Weight in Preadolescents. The J Pediatr 2010;157:911-916.

39. Sherry B, Jefferds ME, Grummer-Strawn M. Accuracy of adolescent self-report of height and weight in assessing overweight status. Arch Pediatr Adolesc Med 2007;161:11541161.

40. Brener ND, McManus T, Galuska DA, Lowry R, Wechsler H. Reliability and validity of self-reported height and weight among high school students. J Adolesc Health 2003;32:281-287.

41. Streiner DL. Starting at the Beginning: An Introduction to Coefficient Alpha and Internal Consistency. J Pers Assess 2003;80:99-103.

42. Pallant J. SPSS survival manual: A step by step guide to data analysis using SPSS for Windows. 3rd ed. Maidenhead: Open University Press; 2007.

43. Lask B, Bryant-Waugh R. (Eds.). Anorexia nervosa and related eating disorders in children and adolescence. 2nd ed. Hove, UK: Psychology Press; 2000. 


\section{APPENDIX A}

\section{Great Ormond Street Criteria ${ }^{43}$}

Food avoidance emotional disorder (FAED)

- Food avoidance not accounted for by primary affective disorders

- Weight loss

- Mood disturbance not meeting criteria for primary affective disorders

- No abnormal cognitions regarding weight or shape

- No morbid preoccupation regarding weight or shape

- No organic brain disease or psychosis

Selective eating (SE)

- Narrow range of foods for at least two years

- Unwillingness to try new foods

- No abnormal cognitions regarding weight or shape

- No fear of choking of vomiting

- Weight may be low, normal, or high

Functional dysphagia (FD)

- Food avoidance

- Fear of swallowing, choking, or vomiting

- No abnormal cognitions regarding weight or shape

- No morbid preoccupation regarding weight or shape 
- No organic brain disease or psychosis

Description of the Eating Disturbances in Youth-Questionnaire (EDY-Q)

The EDY-Q is a screening instrument for assessing early-onset restrictive eating disturbances in middle childhood via self-report. The questionnaire consists of 14 items, two questions of which assess pica and rumination disorders and were therefore excluded in this study. A total of 10 items cover three common early-onset restrictive eating disturbances, with food avoidance emotional disorder (FAED), selective eating (SE), and functional dysphagia (FD). Another two items cover shape and weight concern as important exclusion criteria of these presentations. ${ }^{1}$

\section{Further psychometric properties of the EDY-Q}

A previous study on the psychometric properties of the EDY-Q with the same sample $(N=1444)$ revealed low missing data (0-1.0\%) for each item of the EDY-Q. ${ }^{26}$ Further, item difficulty $\left(.05 \leq p_{m} \leq .38\right)$ was medium to high and corrected item-total correlations $\left(.24 \leq r_{i t}\right.$ $\leq .39$ ) as well as homogeneity of subscale (inter-item correlations: $.19 \leq r \leq .39$ ) were low to medium. Internal consistencies for the four factors were low $(.42 \leq$ Cronbach $\alpha \leq .52)$.

\section{Diagnostic coding of the EDY-Q}

For prevalence estimation on the variants of early-onset restrictive eating disturbances, items were factor-analytically selected for FAED (Interest in Food, Emotional Food Avoidance), SE (Selective Eating Behavior, Sensory Food Avoidance), and FD (Fear of Choking, Fear of Swallowing). The following cut-off criteria were defined: In order to fulfill criteria for FAED, SE, or FD the eating behavior had to be reported at least "often" (cut-off $\geq$ 4). Dichotomization of items (originally scaled 0-6), allowed to code the answers as "no" for scores between 0-3 (“never” to "sometimes”) or as “yes” for scores between 4-6 (“often” to “always”). Distorted cognitions about weight or shape as an exclusion criterion, had to be 
reported less than "sometimes" (cut-off $<3$ ) in order to fulfill the criteria for the variants. An overlap among variants was defined if a child fulfilled the criteria of more than one variant.

Tables

Table 1

Principal component analysis of the EDY-Q items $(N=1444)$

Factors

\begin{tabular}{lllll}
\hline I & II & III & IV & $h^{2}$
\end{tabular}

Rotated factor loadings

Food Avoidance .754

Interest in Food .202

.716

Emotional Food Avoidance

.605

Underweight

.795 .68

Desire to Gain Weight

.812 .69

Selective Eating Behaviour

.567

Avoidance to Try New Foods

.804

Fear of Choking

Fear of Swallowing

$.753 \quad .59$

Sensory Food Avoidance

.715

$.257 \quad .59$

EDY-Q, Eating Disturbances in Youth-Questionnaire (van Dyck et al. 2013); ${ }^{25}$ Principal Component Analysis with orthogonal VARIMAX rotation; Kaiser's normalization; only loadings $\geq .2$ are displayed. 
Table 2

Group differences for variants of early-onset restrictive eating disturbances

\begin{tabular}{|c|c|c|c|c|c|c|}
\hline & \multicolumn{2}{|c|}{ FAED } & \multicolumn{2}{|c|}{$\mathrm{SE}$} & \multicolumn{2}{|c|}{ FD } \\
\hline & $\%$ & $\begin{array}{c}\chi^{2} \\
(d f=1)\end{array}$ & $\%$ & $\begin{array}{c}\chi^{2} \\
(d f=1)\end{array}$ & $\%$ & $\begin{array}{c}\chi^{2} \\
(d f=1)\end{array}$ \\
\hline \multicolumn{7}{|l|}{ Gender } \\
\hline Boys & 20.5 & 0.79 & 30.2 & $9.29^{*}$ & 5.5 & 0.47 \\
\hline Girls & 18.6 & & 23.0 & & 4.7 & \\
\hline \multicolumn{7}{|l|}{ Age } \\
\hline 7-10 years & 23.6 & $10.19^{*}$ & 28.4 & 2.22 & 8.0 & $17.29^{* *}$ \\
\hline $11-13$ years & 16.7 & & 24.9 & & 3.1 & \\
\hline Weight status & & $(d f=2)$ & & $(d f=2)$ & & $(d f=2)$ \\
\hline Underweight & 24.1 & 4.61 & 40.0 & $26.25^{* *}$ & 4.3 & 0.11 \\
\hline Normal weight & 18.2 & & 24.2 & & 4.2 & \\
\hline Overweight & 14.6 & & 14.6 & & 4.9 & \\
\hline
\end{tabular}

FAED, Food Avoidance Emotional Disorder; SE, Selective Eating; FD, Functional Dysphagia; $\chi^{2}$, chi-square test; BMI, body mass index; underweight, $<10^{\text {th }}$ BMI percentile; normal weight, $10^{\text {th }}-90^{\text {th }}$ BMI percentile; overweight, $>90^{\text {th }}$ BMI percentile. ${ }^{*} p<.05 ;^{* *} p<$ .01 
VARIANTS OF EARLY-ONSET 